\title{
Depicting Respiratory Characteristics of Blood Pressure Signal by Using Empirical Mode Decomposition
}

\section{Chia-Chi Chang ${ }^{1,2,3}$, Tzu-Chien Hsiao ${ }^{1,3,4}$ and Hung-Yi Hsu ${ }^{5,6 *}$}

${ }^{1}$ Biomedical Electronics Translational Research Center, National Chiao Tung University, Taiwan, R.O.C

${ }^{2}$ Department of Electronics Engineering and Institute of Electronics, National Chiao Tung University, Taiwan, R.O.C

${ }^{3}$ Institute of Biomedical Engineering, National Chiao Tung University, Taiwan, R.O.C

${ }^{4}$ Department of Computer Science, National Chiao Tung University, Taiwan, R.O.C

${ }^{5}$ Department of Neurology, School of Medicine, Chung Shan Medical University, Taichung, Taiwan, R.O.C

${ }^{6}$ Section of Neurology, Department of Internal Medicine, Tungs' Taichung Metro Harbor Hospital, Taichung, Taiwan, R.O.C

\begin{abstract}
Aim: To explore adequate parameters for EMD of ABP signal; to determine the intrinsic characteristics of $A B P$ waveform through the analysis of IMFs' averaged period and its energy density; to examine the effect of different respiration patterns on IMFs extracted from ABP waveform by CEEMD.

Arterial blood pressure (ABP) reflects cardiac function, vessel compliance, and cardiorespiratory interaction and $A B P$ analysis provides the estimators of this physiological information. But it is inconvenient for quantitative $A B P$ assessment due to several influences, such as respiration. Recently, a novel adaptive method, called empirical mode decomposition (EMD), was proposed, and it was useful for non-stationary intrinsic characteristics extraction. Though some literatures examined that EMD helps for physiological signal analysis study, the method applied for ABP signal still needs further investigation. This study proposed a standard procedure of specific EMD for ABP intrinsic characterization during spontaneous breathing, 6-cycle breathing, and hyperventilation. The extracted components, called intrinsic mode functions (IMFs), were determined with the examined parameters, including ensemble number, added noise, and the stop criterion. The IMFs of ABP signal were categorized into five major intrinsic components, including the noise and irregular fluctuation (IMF1), beat-to-beat cardiac intervals (IMF2), characteristics of pressure waveform morphology (IMF3), base beat (IMF4), and respiratory related fluctuation (IMF5 and IMF6).

The results showd that the characteristics of IMFs were quantified by averaged period and corresponding energy density with good reproducibility. The proposed algorithm produced meaningful IMFs representing the cardiac rhythm, intrinsic waveform mophology, and the intrinsic influence of respiration fluctuations. EMD helps for analyzing the underlying mechanisms of control processes, including cardiorespiratory coupling and interactions among organ systems at multiple time scales.
\end{abstract}

Keywords: Arterial blood pressure; Respiration; Intrinsic mode function; Empirical mode decomposition; Hilbert-Huang transform

\section{Introduction}

Arterial blood pressure (ABP) is one of the most important parameters of systemic circulation. ABP is principally determined by cardiac pumping function and resistance of peripheral vessels, which controlled by autonomic nerve system. Non-invasive ABP waveform has been used to estimate some physiological indices, such as cardiac output, peripheral resistance and arterial compliance [1,2]. The ABP waveform consists of the forward pressure wave generated by the heart and the backward reflection wave reflected back by the branches of vessels [2,3]. The velocity of the reflection pressure wave, which contributes to the morphology of ABP waveform, is an indicator of arterial compliance [1,2]. Analysis of morphologic indices of $\mathrm{ABP}$ waveform, such as systolic peaks, diastolic valleys, dicrotic notches, and augmented index, have been used to evaluate the activities of autonomic nerve system [4,5], arterial stiffness [2,3], cardiac function [6] and detection of cardiac arrhythmia [7]. However, most of these algorithms are only suitable for certain physiological states [8,9].

The ABP usually fluctuates in long-term monitoring. Respiratory movement causes the changes of $\mathrm{ABP}$ and corresponding changes of heart rate (HR). The effect of respiration on $\mathrm{HR}$, named respiratory sinus arrhythmia, is considered as a surrogate of cardiac vagally modulation. Respiratory sinus arrhythmia has been widely applied in the calculation of spontaneous baroreflex sensitivity [10], though the precise phase dependency remains unclear. On the other hand, the extent of $\mathrm{ABP}$ fluctuation during respiration is related to central blood volume and cardiac-pulmonary coupling [11]. The regulation relationship between respiratory movement, BP and HR had been studied by using the fast Fourier transform (FFT) or discrete Wavelet transform (DWT) as frequency domain analysis [12]. FFT and DWT had been used for waveform decomposition in time-frequency characteristics researches, including heart rate variability [13] and blood pressure variability $[12,14,15]$, analysis. However, these well-known transforms caused signal distortion and information loss due to the limitation caused by fundamental assumption, such as sinusoidal linear combination in FFT or discrete wavelet function in DWT. In recent years, Hilbert-Huang transform (HHT) [16] has been used increasingly in data processing researches because of its adaptive capability on non-stationary and non-linear decomposition. The distortion of original signals was controlled and limited, because HHT is fundamentally based on the entire morphology of waveform [16]. HHT may help to specify slow or non-rhythmic fluctuation of ABP.

The capability of HHT in dealing with non-linear and non-

*Corresponding author: Hung-Yi Hsu, Department of Neurology, Tungs' Taichung Metro Harbor Hospital, No. 699, Sec. 1, Jhongci Road, Wuci District, Taichung 435, Taiwan, R.O.C, Tel: +886-9-10597960; Fax: +886-4-26582193; E-mail: hungyihsu@gmail.com

Received July 08, 2014; Accepted October 20, 2014; Published October 24 2014

Citation: Chang CC, Hsiao TC, Hsu HY (2014) Depicting Respiratory Characteristics of Blood Pressure Signal by Using Empirical Mode Decomposition. J Pulm Respir Med 4: 209. doi:10.4172/2161-105X.1000209

Copyright: ( $) 2014$ Chang CC, et al. This is an open-access article distributed under the terms of the Creative Commons Attribution License, which permits unrestricted use, distribution, and reproduction in any medium, provided the original author and source are credited. 
stationary signal comes from essential pre-process, called empirical mode decomposition (EMD). The EMD, pioneered by N.E. Huang et al, decomposes non-linear and non-stationary time series into finite set of mono-components, called intrinsic mode functions (IMFs) [17]. The EMD extracts the IMFs iteratively by detrending operation, called sifting process, based on the morphology of waveform. The essence of sifting process is based on iteratively energy associated extraction at each time scale. The EMD has been applied on HHT as a new timefrequency analysis method in various applications [18]. Recently, Huang et al. characterized white noise by the spectral energy density and the averaged period [19]. This method could qualitatively and quantitatively evaluate the intrinsic characteristics of non-linear and non-stationary signal. This method has potential to characterize the intrinsic feature of $\mathrm{ABP}$ waveform.

The EMD is able to reduce noise as a filter bank [20-22]. But the EMD decomposition process contains the intermittency phenomenon, called mode mixing problem, owing to the sensitivity of cubic spline interpolation and end effects [16]. The mode mixing problem would lead to the transitional gaps in further examination with Hilbert transform. In order to overcome mode mixing problem, a noise-assisted data analysis method, known as ensemble EMD (EEMD), was proposed [23]. The EEMD helps to eliminating the mode mixing problem by repeated adding white noise of specific standard deviation. The final set of ensemble IMFs is obtained by averaging each corresponding IMF decomposed from different mixtures. The EEMD has been used in several medical studies [24-27], though EEMD is insufficient for characterization of ABP signal owing to its non-zero residue. The residue of added white noise could be removed by adding positive and negative white noises respectively as two mixtures of the source signal and added white noise, which is called complementary EEMD (CEEMD). The CEEMD not only extends the concept of EEMD but also eliminates the residue of added white noises. The characteristics of IMFs extracted by CEEMD are much stable [28]. However, the IMFs extracted by CEEMD are affected by the parameters used in CEEMD, such as ensemble number, added white noise, and stop criterion of IMF determination. The optimal setting for CEEMD applying on $\mathrm{ABP}$ analysis has not been standardized.

\section{Objectives and Methods}

\section{Subjects and data collection}

All measurements were performed in a quiet temperaturecontrolled room. The beat-to-beat ABP waveform was recorded by Task Force ${ }^{\infty}$ Monitor equipped with a servo-controlled plethysmography (CNSystems, Medizintechnik AG, Graz, Austria). Electrocardiography (ECG), thoracic impedance and end-tidal $\mathrm{CO}_{2}$ concentration $\left(\mathrm{EtCO}_{2}\right.$; RespSenseTM EtCO ${ }_{2}$, Nonin Medical Inc., Ply-mouth, Minnesota, USA) were recorded simultaneously. The signals of ABP, ECG and thoracic impedance were recorded and stored by the CNSystem. The analog signal of $\mathrm{EtCO}_{2}$ was fed via an analog-input interface to the CNSystem at a sampling rate of $200 \mathrm{~Hz}$, and saved on the hard disc. All data were exported to a computer for later analysis.

Ten subjects (age $47 \pm 21.5$ male) participated in this study. All recruited subjects were asked to rest quietly in supine position for 10 minutes. After all recorded signals were stabilized, a 10-min baseline recording was performed under spontaneous breathing. Subsequently, recruited subjects were asked to breathe following the examiner's verbal instruction at a rate of 6 cycles per minute for 2 minutes. After a 5-minute rest, subjects were asked to perform hyperventilation at a rate of 30 cycles per minute for 2 minutes. This study was approved by institutional review board of the hospital. Informed consent was obtained from all subjects before the experiment.

\section{Ensemble empirical mode decomposition}

The algorithm of EMD contains several steps. First, the local extrema in the time series $x(t)$ were identified by peak-valley detection. The upper envelope $L(t)$ and lower envelope $L(t)$ were calculated by cubic spline interpolation based on the local maxima and the local minima. Both envelopes covered $x(t)$ at each time scale. The trend waas estimated by the average of $U(t)$ and $L(t)$.

$$
m(t)=(U(t)+L(t) / 2
$$

The first intermidiate component $h_{1}(t)$ was extracted by subtracting the trend from the original time series $x(t)$, as the detrending process.

$h_{1}(t)=x(t)-m_{1}(t)$

The subtraction was performed iteratively until the trend satisfied the predetermined criterion. The predetermined criterion is adopted the value of the standard deviation of the trend ${ }^{m_{1}}(t)$, which was seen as a steady constant trend while the value is below 0.2 .

$$
\left\{\begin{array}{c}
h_{1}(t)=x(t) \\
h_{1 k}(t)=h_{1(k-1)}(t)-m_{1 k}(t) k>1
\end{array}\right.
$$

$h_{1 k}(t)$ would be considered as an IMF only if the trend satisfies the criterion after $\mathrm{k}$ times of the subtraction process.

$$
h_{1 k}(t)=I M F_{1}(t)
$$

The first residue was calculated by the equation (5).

$$
r_{1}(t)=x(t)-I M F_{1}(t)
$$

The residue ${ }^{r_{i}(t)}$ was the target signal of ith iteration of EMD for the ith component extraction. After $\mathrm{n}$ iteration, $x(t)$ was decomposed into $\mathrm{n} \mathrm{IMFs,} I M F_{1}(t) \sim I M F_{n}(t)$, and one residue, ${ }_{n}{ }^{(t)}$, which can be either the steady trend or a constant.

$$
x(t)=\sum_{i=1}^{n} I M F_{i}(t)+r_{n}(t)
$$

EMD decomposes the non-stationary signal into a finite set of IMFs without information loss or distortion, and the results were refined by EEMD and much further refined by CEEMD, which added white noise into the source signal before EMD and got the final IMFs by average the IMFs corresponding to the added white noise. The added times of the white noise were called ensemble number and presented the times of EMD. This study adopted CEEMD for the ABP waveform decomposition.

According to the noise dependency of noise-assisted data analysis method, the variation of analysis results with different white noise would be considered and verified at first. For eliminating the noise effect of CEEMD, different processing parameters for CEEMD including the magnitude of white noise, ensemble number, and stop criteria were tested. Adequate setting parameters used in this study was selected by the consistency of IMF features in different physiological situation, including baseline, 6-cycle breathing and hyperventilation. The reproducibility of the characteristics of IMFs depends on its variation between repeated analyses. The reproducibility of the decomposition results was evaluated by time sliding window variation with 10 trails. The result of CEEMD was stabilized while the ensemble number, which presents the times of ensemble iteration with added white noise, is greater than 200. The results suggested that 200 ensemble number is enough for ABP signal analysis. The analysis system used in this study was developed by using a commercial programming platform (LabVIEW, version 2011, National Instruments Corp., Austin, TX, USA). 


\section{Determination of intrinsic mode function}

The IMF is the function that satisfies the condition that the mean of the upper envelope and lower envelope, as defined by local extrema, approximates zero at any time instant [29]. However, the mean envelope is hard to achieve zero because of the non-stationary signal interference. There were several stop criteria for IMF determination, such as standard deviation, $S$ number criterion, energy difference tracking or amplitude ratio [30]. Based on different stop criterion, the IMF decomposed from source signal would be different with various fundamental definitions. This study applied the direct standard deviation of mean envelope as the stop criterion rather than the standard deviation of the division of mean envelope by the intermediate signal. The IMF extraction would be faster than the original EMD stop criterion and well controlled due to its quick convergence.

\section{Averaged period and energy density}

The CEEMD technique is usually utilized for Hilbert transform pre-process as the whole process of HHT and for mono-component extraction in order to avoid the negative frequency effect [16]. However, the marginal spectrum of HHT spectrum has the effect of precise characterization, called intra-wave frequency modulation, and it makes non-stationary quantitative evaluation much difficult in specific circumstance, such as all physiologic challenges. The characteristics of each IMF as non-stationary mono-component could be defined by the distribution of energy density and its corresponding averaged period. This method was originally used to study the characterization of white noise by EMD and to evaluate the distribution of energy density and corresponding averaged period of white noise [19].

$$
\begin{aligned}
& E_{n}=\frac{1}{N} \sum_{t=1}^{N}\left[I M F_{n}(t]^{2}\right. \\
& \bar{T}_{n}=\int S_{\mathrm{h} T, n} d \mathbf{h} T\left(\int S_{\mathrm{h} T, n} \frac{d \mathrm{~h} T}{T}\right)^{-1}
\end{aligned}
$$

$E_{n}$ is the energy density of the nth IMF and $S_{\mathrm{h} T, n}$ is the Fourier spectrum of the nth IMF as the function of $\mathrm{h} T . T$ is the period along the axis of the spectrum after changing variable, and ${ }^{T_{n}}$ is the estimator of the averaged period of the nth IMF.

The characterization of white noise IMFs was verified by Monte Carlo method, which generated 1000 different white noise and decomposed each of them in order to examine the confidence interval of the distribution of the intrinsic noise component [19]. The sum of logarithmic averaged period $\mathrm{h} \bar{T}_{n}$ and logarithmic energy density $\mathrm{h} E_{n}$ would approximate to a constant [31]. The averaged period and energy density had potential to demonstrate the characteristics of IMFs extracting from non-stationary source signal by EMD. In this study, this method was applied to determine the characteristics of IMFs.

\section{Results}

\section{Selection of white noise}

The results of noise-assisted data analysis, such as CEEMD, would vary when different white noises are used. This study tested different added white noises in CEEMD and the results are shown in Figure 1. The mode mixing problem is obviously in the result of EMD in Figure 1a and is markedly attenuated by CEEMD. The IMFs are well discriminated along with increasing standard deviation of added white noise. The characterization of IMFs by averaged period and energy

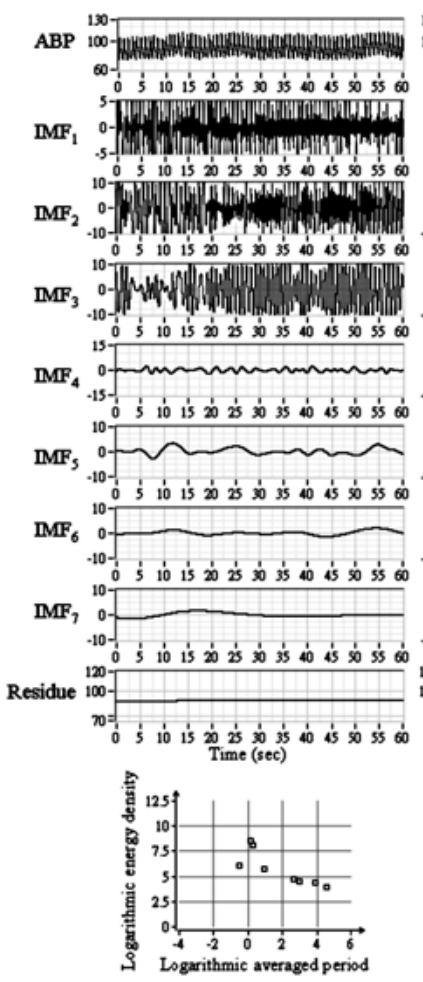

(a)

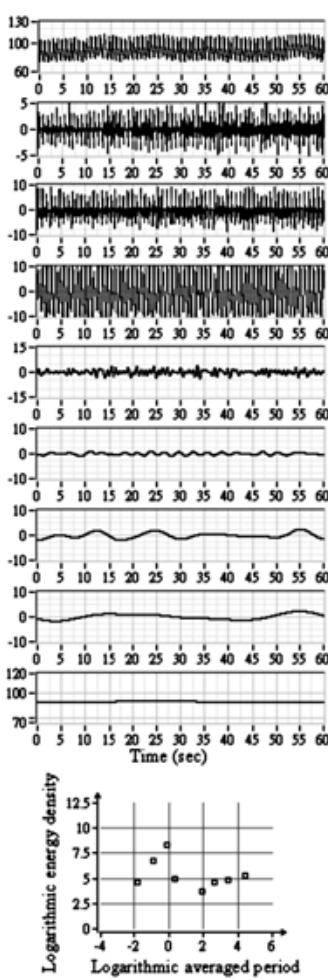

(b)

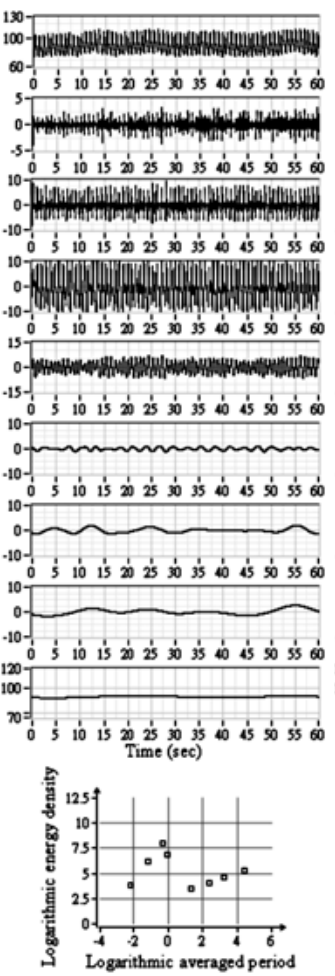

(c)

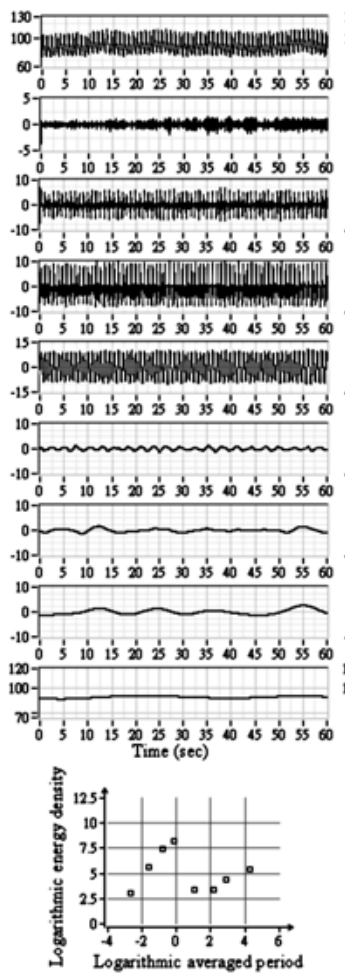

(d)

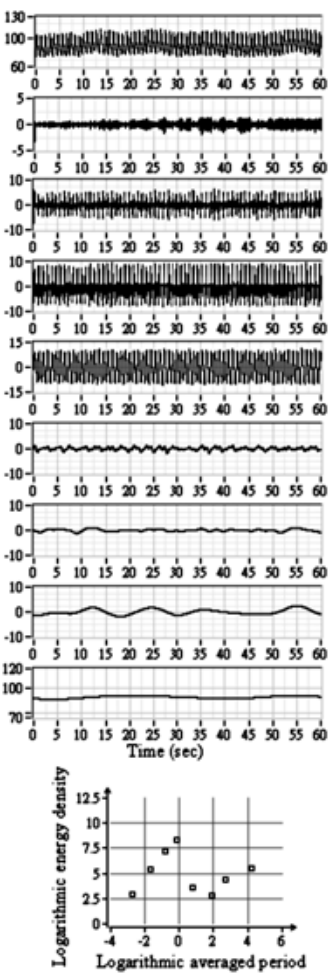

(e)

Figure 1: Results of EMD and CEEMD for ABP signal with different added noise. In all panels, top graph presents the original ABP signal, IMF1-IMF7 and the residue are listed below, and the bottom graph is the characterization plot with logarithmic averaged period and logarithmic energy density of each IMF. (a) EMD of ABP signal, CEEMD with (b) 0.1 , (c) 0.2 , (d) $1 / 15$ time of standard deviation (SD) of original signal, and (e) 1/10 time of SD of original signal as the SD of noise. 
density was well separated without overlapping in noise-assisted analysis. High-frequency and irregular fluctuation appears in IMF5 in Figure 1e when the standard deviation of added white noise was greater than one-tenth of standard deviation of source signal. According to these observations, one-fifteenth of standard deviation of source signal as the standard deviation of noise was selected as the setting of added white noise for ABP signal decomposition in CEEMD in this study.

\section{Reproducibility analysis}

Ten 1-minute segments of resting $A B P$ signal from each subject was sampled and decomposed by CEEMD respectively. The characteristics of IMFs were similar within the same individual. The coefficient of variation (CV) of logarithmic averaged period and the $\mathrm{CV}$ of logarithmic energy density in each subject were calculated for the reproducibility verification. Figure 2 shows that the $\mathrm{CV}$ of logarithmic averaged period and the $\mathrm{CV}$ of logarithmic energy density are small. The CV of logarithmic averaged period of IMF4 is relatively high owing to its mean approaching zero. The CV of both the logarithmic averaged period and energy density were high in IMF5 and IMF6 because of variable respiration-related fluctuation of ABP between subjects (further discussion in next paragraph). The results suggested that the methods used for characterization of IMFs in this study were reliable. Even though the ABP signal was recorded at different time points, the CEEMD of ABP waveform had good reproducibility in different individuals

\section{Characteristics of IMFs during different breathing patterns}

The results of CEEMD decomposition of ABP signals during different breathing patterns are shown in Figure 3. The characteristics of IMFs during spontaneous breathing, 6-cycle breathing, and hyperventilation were similar. The IMFs derived from CEEMD decomposition of ABP signal were categorized into five major components, including noise, beat-to-beat time intervals, characteristics of pressure waveform, base beat, and respiratory related fluctuation with the same setting of CEEMD. The IMF1 derived from CEEMD of ABP signal shows the most high frequency component in $\mathrm{ABP}$, which may contain intrinsic characteristics of white noise with orther unexpected fluctuations demonstrated in Figure 4. The IMF2 shows peak-to-peak pressure pulse waveform as shown in Figure 5. Figure 5 shows that the morphology of $\mathrm{ABP}$ waveform was demonstrated by the first four IMFs in a small time scale, and the influence of respiration was eliminated from these four
IMFs. The IMF5 and IMF6 associate with respiration related fluctuation of ABP, as shown in Figure 3, and present the influence of respiration, which was extracted from the orignal ABP signal and was eliminated from the other IMFs. The results of the logarithmic averaged period and corresponding logarithmic energy density were summarized in Table 1.

\section{Beat-to-beat time intervals extracted from ABP signal}

The IMF1 is within the confidence interval of energy density and averaged period of white noise by Monte Carlo verification as shown in Figure 4. The IMF1 represented the most high frequency component in ABP signal, which was more likely to be contaminated by noise caused by some artifacts or the other external influences in Figure $5 \mathrm{~b}$. IMF2 presents the sharpest changes of beat-to-beat $A B P$ and could be a surrogate to determine the beat-to-beat time intervals which is derived from ECG recording conventionally. The beat-to-beat time series were calculated based on IMF2, as shown in Figure 6. The automatic peak detection was applied for first order difference of IMF2 and the time intervals were calculated by peak-to-peak duration, as described in the literature study [8]. Then, the time series were generated from the time intervals by spline interpolation, as an approach to get the beatto-beat time intervals. The interclass correlation test showed excellent correlation between beat-to-beat time intervals extracted from ABP signal and beat-to-beat cardiac intervals from ECG recording during baseline, 6-cycle breathing and hyperventilation (all interclass correlation coefficient $>0.980$ in study subjects).

\section{Characteristics of $\mathrm{ABP}$ waveform morphology}

Figure $5 \mathrm{a}$ shows that the amplitude of IMF3 was larger when the ABP waveform was sharper, while in Figure $5 c$, the amplitude of IMF3 was smaller when the ABP waveform was flat. Similarly, the averaged period and energy density of IMF3 are different when the shape of ABP waveform varies between different individuals, as shown in Figure 5. The IMF4 represents the fundamental base beat waveform as the ABP waveform carrier, and its amplitude and frequency can indicate the related characteristics of $\mathrm{ABP}$ waveform. The characterization plot shows that IMF4 has the largest energy density in Figure 5. The logarithmic averaged period of IMF4 is approximately zero due to the beat-to-beat time period round about 1 second in normal subjects.

\section{Respiration related fluctuations of ABP Signal}

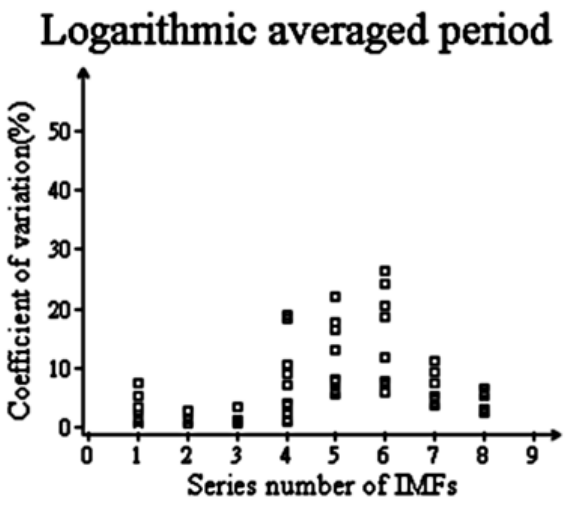

(a)

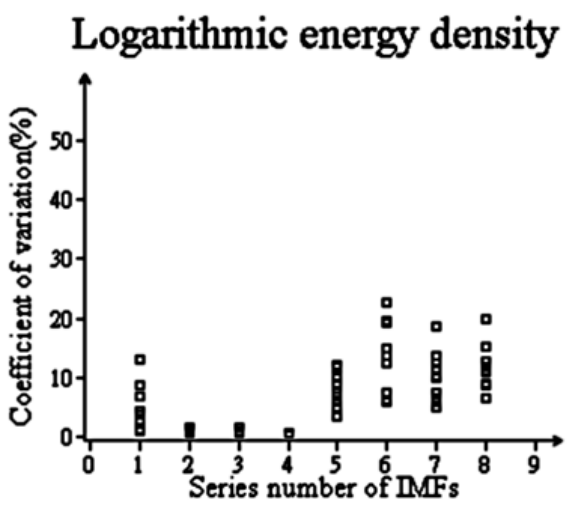

(b)

Figure 2: The coefficient of variation (CV) of (a) logarithmic averaged period and of (b) logarithmic energy density in all subjects for each IMF, series number 1-7 present the number of IMF and 8 presents the residue of CEEMD. 


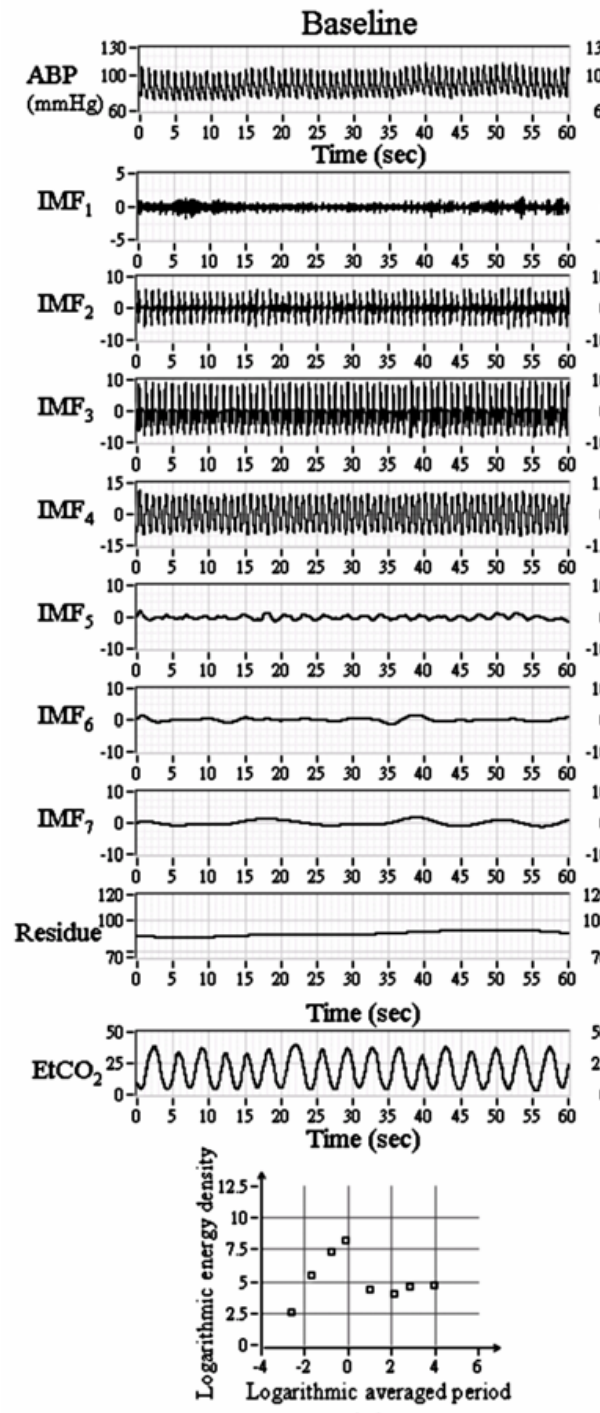

(a)

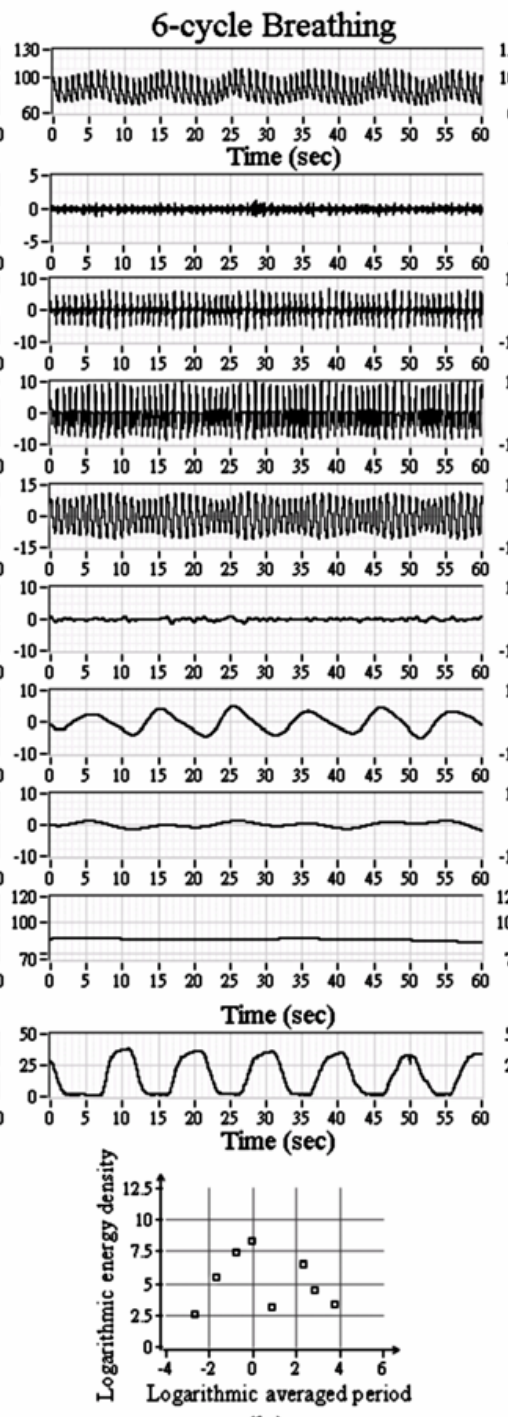

(b)

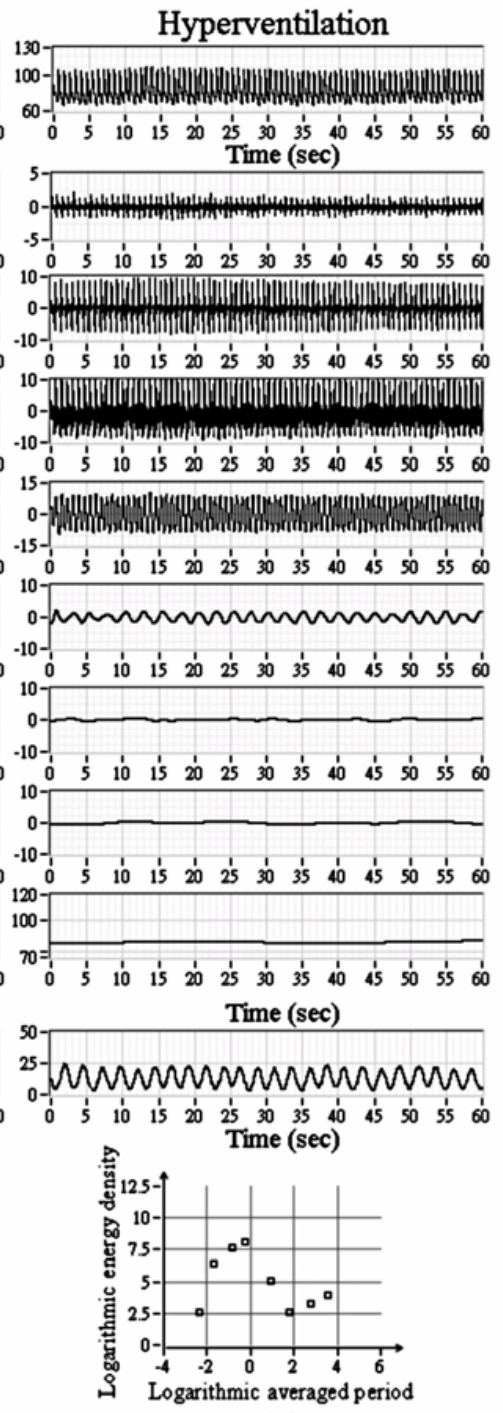

(c)

Figure 3: Results of CEEMD for ABP signal during baseline, 6-cycle breathing and hyperventilation. All panels represent $C E E M D$ of $A B P$ signal, where top graph presents the original ABP signal, IMF1-IMF7 and residue after IMF extracted are listing below, and bottom graph is the corresponding recording of end-tidal $\mathrm{CO}_{2}$ (EtCO ${ }_{2}$ ) concentration. (a) baseline resting spontaneous respiration; (b) during paced breathing at 6 cycles per minute. (c) during hyperventilation at 30 cycles per minute.

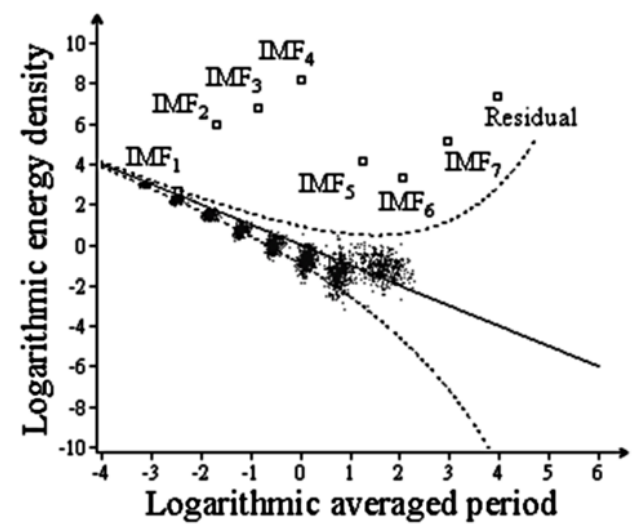

Figure 4: Monte Carlo verification of the relation between the logarithmic averaged period and the logarithmic energy density of IMFs (empty squares with labels) and noise (clusters of dots) 


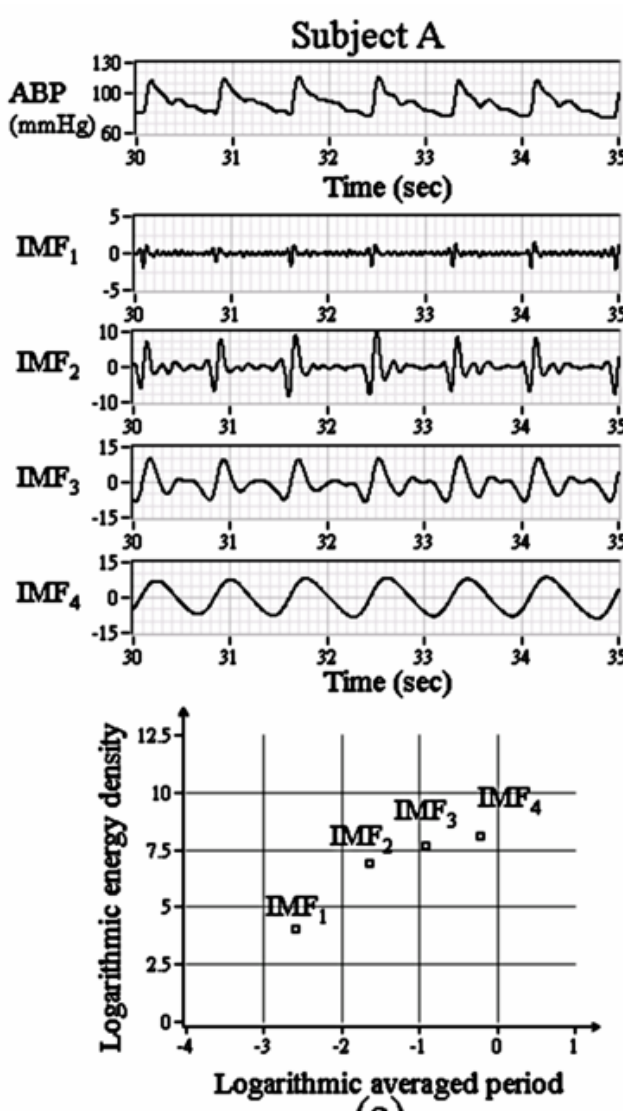

(a)
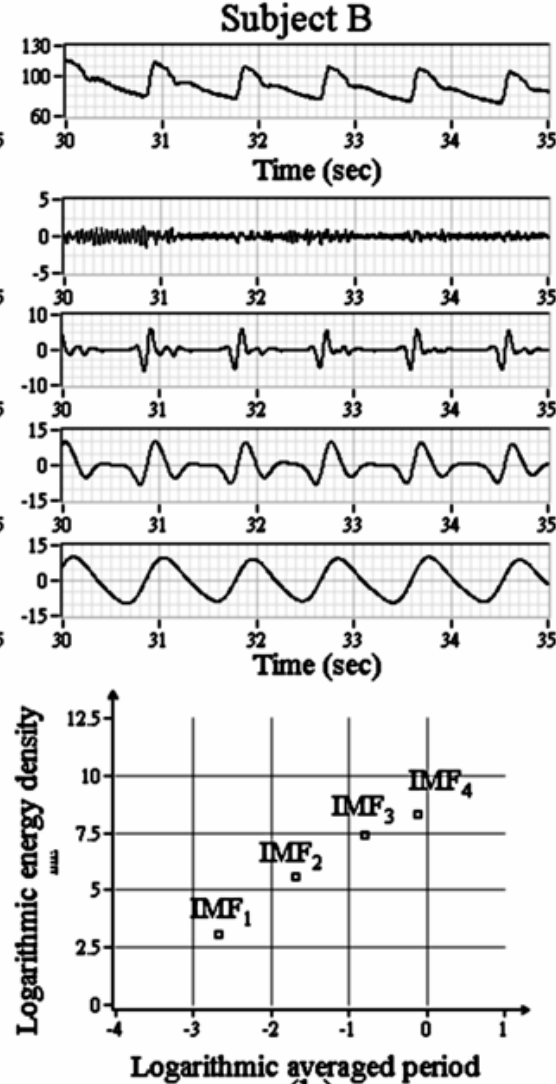

(b)
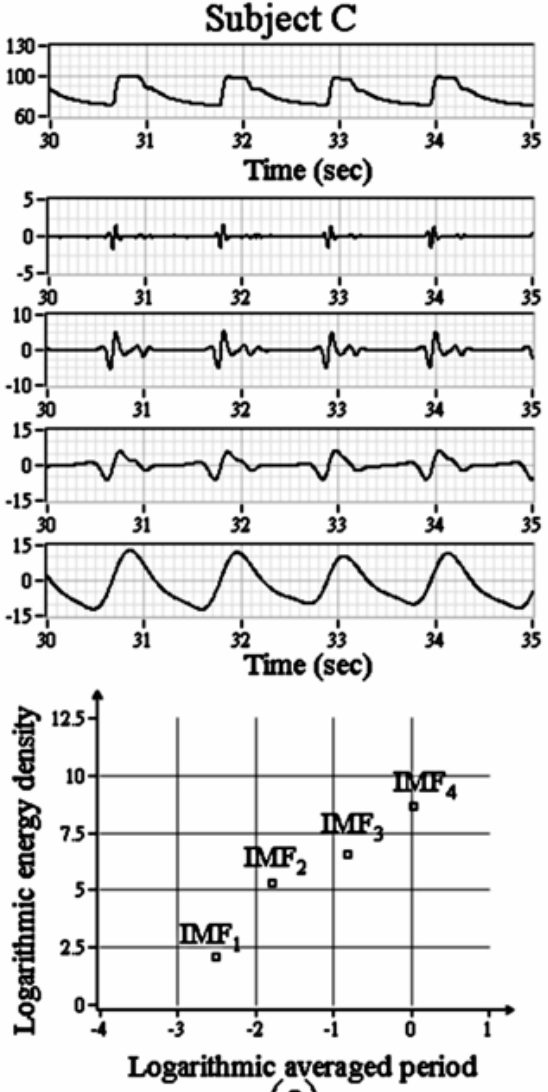

(c)

Figure 5: Results of CEEMD for different $A B P$ waveform morphology in three different subjects. All panels represent CEEMD of $A B P$ signal, where top graph presents the original ABP signal, IMF1-IMF4 are listing below, and the bottom graph is the characterization plot with logarithmic averaged period and logarithmic energy density of each IMF.

\begin{tabular}{|c|c|c|c|c|c|c|}
\hline & \multicolumn{2}{|c|}{ Baseline } & \multicolumn{2}{|c|}{ 6-cycle Breathing } & \multicolumn{2}{|c|}{ Hyperventilation } \\
\hline & $\ln T$ & InE & $\ln T$ & $\ln E$ & $\ln T$ & InE \\
\hline IMF1 & $-2.43 \pm 0.13$ & $2.4 \pm 0.76$ & $-2.44 \pm 0.15$ & $2.17 \pm 1.04$ & $-2.47 \pm 0.21$ & $2.58 \pm 0.9$ \\
\hline IMF2 & $-1.67 \pm 0.12$ & $5.96 \pm 0.51$ & $-1.66 \pm 0.11$ & $5.91 \pm 0.68$ & $-1.62 \pm 0.11$ & $6.09 \pm 0.55$ \\
\hline IMF3 & $-0.85 \pm 0.09$ & $7.39 \pm 0.51$ & $-0.85 \pm 0.08$ & $7.35 \pm 0.6$ & $-0.88 \pm 0.09$ & $7.63 \pm 0.47$ \\
\hline IMF4 & $-0.16 \pm 0.11$ & $8.33 \pm 0.73$ & $-0.12 \pm 0.12$ & $8.31 \pm 0.74$ & $-0.23 \pm .012$ & $8.25 \pm 0.72$ \\
\hline IMF5 & $0.92 \pm 0.26$ & $3.79 \pm 0.79$ & $0.84 \pm 0.2$ & $3.75 \pm 0.62$ & $0.93 \pm 0.17$ & $4.91 \pm 0.61$ \\
\hline IMF6 & $1.85 \pm 0.21$ & $3.24 \pm 0.7$ & $2.27 \pm 0.19$ & $5.35 \pm 1.07$ & $1.86 \pm 0.27$ & $3.49 \pm 1.52$ \\
\hline IMF7 & $2.84 \pm 0.2$ & $3.45 \pm 1.1$ & $2.77 \pm 0.24$ & $4.86 \pm 1.03$ & $2.91 \pm 0.26$ & $3.68 \pm 1.52$ \\
\hline Residue & $3.57 \pm 0.2$ & $2.69 \pm 1.15$ & $3.65 \pm 0.2$ & $3.45 \pm 1.45$ & $3.58 \pm 0.19$ & $2.98 \pm 1.43$ \\
\hline
\end{tabular}

The presented form is (average \pm standard deviation). IMF denotes intrinsic mode function; InT denotes logarithmic averaged period; InE denotes logarithmic energy density. Table 1: The logarithmic averaged period and corresponding logarithmic energy density of each IMF during different breathing patterns.

The respiration-related fluctuation of ABP signal was shown in IMF5 or IMF6, as shown in Figure 3. The $\mathrm{EtCO}_{2}$ concentration was highly concordant with the IMF6 during slow 6-cycle breathing and with IMF5 during rapid hyperventilation. The Bland-Altman plot showed good agreement between the logarithmic averaged period with IMF6 of ABP signal and EtCO concentration during 6-cycle breathing, shown in Figure 7. The logarithmic averaged period with IMF5 of $\mathrm{ABP}$ signal also had good agreement with $\mathrm{EtCO}_{2}$ concentration during hyperventilation. The phase of ABP fluctuation had a reversal relationship with that of $\mathrm{EtCO}_{2}$ concentration in different respiratory patterns. The amplitude change of respiration signal correlated with the energy density of corresponding IMF. The results in Table 1 showed that the logarithmic averaged period provides the estimation of the relevant main period of each IMFs. The energy density of IMF6 increased during 6-cycle breathing and the energy density of IMF5 increased during hyperventilation.

\section{Discussion}

In general, CEEMD has good capability for non-stationary signal decomposition, but its result depends on the setting of several parameters. The proper selection of added white noise of CEEMD and the proper stop criterion of IMF determination are important to characterize the ABP signal [32]. The study suggested that CEEMD of ABP signal produces reliable results by using 1/15 time of standard 


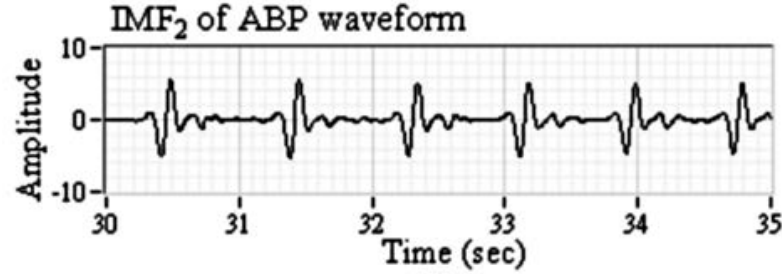

(a)

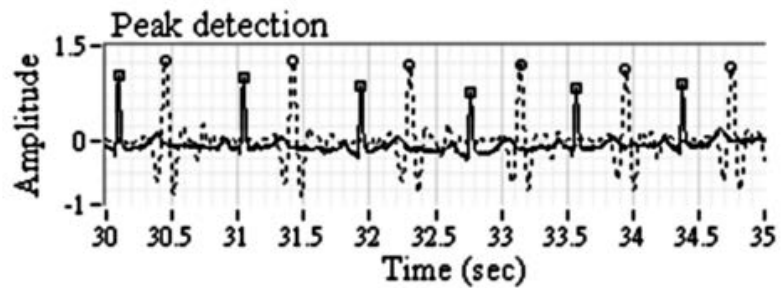

(b)

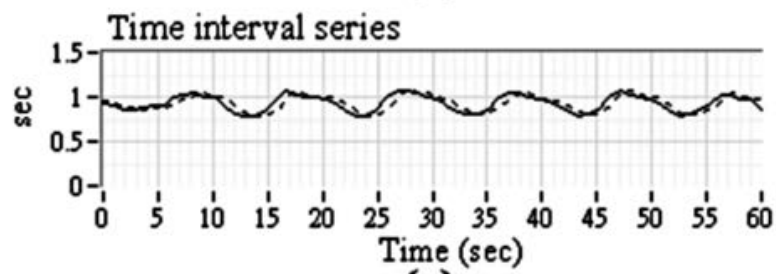

(c)

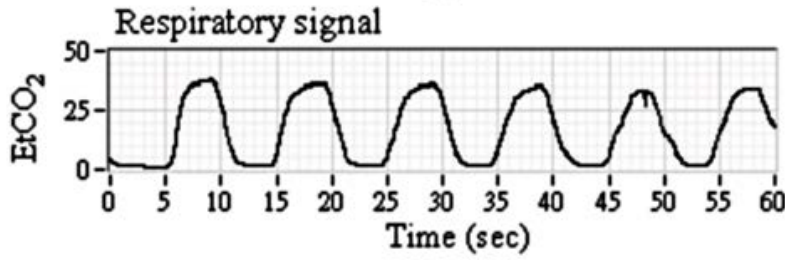

(d)

Figure 6: An illustration of beat-to-beat cardiac interval extracting from the IMF2 of ABP signal and relationship with respiration. (a) the original IMF2 of ABP signal, (b) the peak detection of R-wave in ECG (solid line) and that of first order difference of IMF2 (dotted line), (c) the RR intervals series in ECG (solid line) and the beat-to-beat time interval series from IMF2 (dotted line), and (d) the $\mathrm{EtCO}_{2}$ concentration.

deviation of ABP source signal as the standard deviation of added noise of CEEMD and directly referred standard deviation of intermediate signal as the stop criterion for ABP signal characterization. The optimal parameters of CEEMD analysis would vary when different physiological signals are the targets of CEEMD. This study demonstrated the process to achieve reliable analysis.

CEEMD is able to extract important physiologic and pathologic information from ABP signal. The sifting process gives CEEMD ability to discriminate non-stationary orthogonal characteristics of morphology, such as frequency, in various time scales. Each IMF decomposed by CEEMD represents a frequency-amplitude modulation in narrow frequency band that can be related to a specific physiologic process [33]. However, most of previous studies applied EMD in analysis of systolic BP tachogram only [34], or just focused on the characteristics of one or two IMFs [27,34]. This study demonstrated the important features of all IMFs and their possible physiological meanings, which were verified by multi-modality monitoring and performing different respiratory maneuvers. By using CEEMD, the ABP signal can be decomposed into meaningful IMFs representing cardiac intervals, characteristics of pressure waveform, and respiratory pattern in this study.

The first four IMFs were regarded as non-physiological interferences owing to its frequency range and contained rich information of intrinsic waveform mophology. By the adequate parameters setting of CEEMD, the non-physiological artifacts and noises were restricted within IMF1. The CEEMD effectively eliminated artifacts in the other IMFs. Thus, the beat-to-beat cardiac interval and the mophology analysis, such as reflection wave analysis, were estimated accurately by further processing without the other unexpected influences.

The morphology of ABP waveform is the summation of forward pressure wave and backward reflection wave, and is considered as the characteristics of vessel compliance referred to the reflection coefficient [35]. Certain IMFs, for example IMF3 and IMF4 in this study, preserved the features of original ABP waveform morphology. Some indices of arterial stiffness and atherosclerosis could be derived from these IMFs [27,34]. Further post-processing to characterize subtle changes in these IMFs may have potential to be used in classification of ABP waveform for pulse diagnosis [36] and cardiovascular researches.

The respiratory influence of ABP signal was observed in IMF5 or/ and IMF6 in different respiratory patterns. The adaptive sifting process of CEEMD, which sifting the signal iteratively from high frequency to low frequency in various time scale, distributes the respirationrelated ABP changes to different IMF depending on the frequency of respiration. The phenomenon was quantitative assessed by the averaged period, shown in Table 1 . The energy density of IMF6 increased during 6-cycle breathing and the corresponding logarithmic averaged period was 2.27 , which means that the averaged period was $9.68 \mathrm{sec}(\sim 10$ $\mathrm{sec}$ ) and it met the period of 6-cycle breathing. On the other hand, the energy density of IMF5 increased during hyperventilation and the corresponding logarithmic averaged period was 0.93 , which means that the averaged period was $2.53 \mathrm{sec}(\sim 10 \mathrm{sec})$ and it met the period of hyperventilation. The good agreement between logarithmic averaged period of $\mathrm{EtCO}_{2}$ and that of the corresponding IMF suggested that respiratory pattern could be evaluated by CEEMD of ABP signal. By using CEEMD, ABP recording not only provided hemodynamic information, but also had potential to detect abnormal respiratory pattern such as sleep apnea. The ABP change had a reversal relationship with $\mathrm{EtCO}_{2}$ concentration in different respiratory patterns $[37,38]$. The

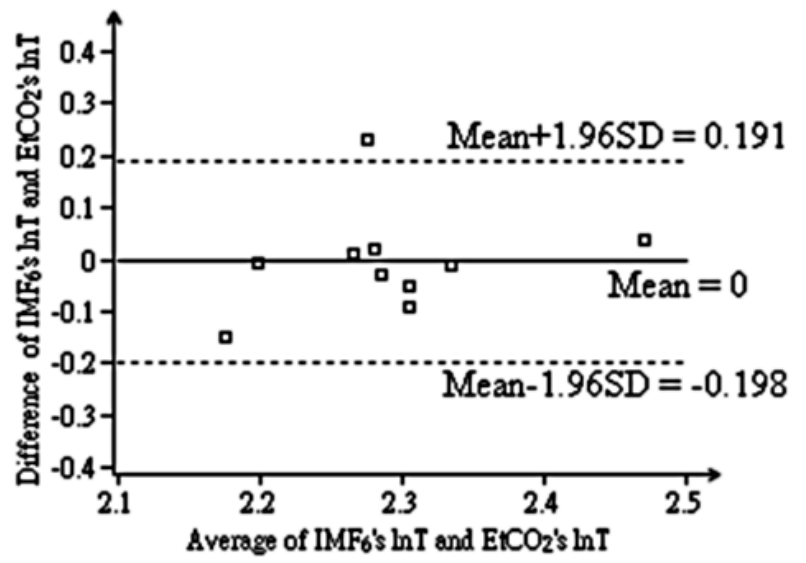

Figure 7: Agreement between logarithmic averaged period of IMF6 of ABP signal and that of $\mathrm{EtCO}_{2}$ during 6-cycle breathing by using Bland-Altman plot. The mean difference and the limits of agreement are indicated. 
phase delay between $\mathrm{EtCO}_{2}$ signal and respiratory fluctuation of $\mathrm{ABP}$ signal was similar to previous reports [39].

The CEEMD had good capability of non-stationary sifting and was served as the time-variant filter bank adaptively in various time scales. The IMF decomposed by CEEMD represents a posteriori monocomponent in narrow frequency band, which might be related to a specific physiologic process, and it might help the physiological study finding the optimal frequency band of regulation in multiple time scale. The IMF7 and the following IMFs have the potential to explore the very low frequency band $(<0.04 \mathrm{~Hz})$, i.e. high logarithmic averaged period (>3.2) on characterization plot of IMF, in long-term ABP monitoring. Cardiovascular regulation in this frequency range may result from the metabolic, humoral, or temperature modulation [5]. The EMD decomposition of short-term ABP recording is not able to evaluate these low frequency changes.

This study proposed a simple method to quantitatively evaluate the non-stationary characteristics of real medical signal, ABP for example, by using averaged period and energy density. These two parameters contained the morphology information extracted by CEEMD, and quantified the morphology information based on spectral integration. The averaged period and energy density had good reproducibility on repeated measurements in this study. However, these two parameters could only depict the differences in $\mathrm{ABP}$ waveform morphology roughly.

This study has some limitations. First, the resolution of the averaged period is limited by the sampling rate of the original source signal, and the bound of averaged period is determined by the recording period. Secondly, the characteristics of IMFs are mainly determined by the parameters of CEEMD. It seriously limits the IMF usefulness in characterization. The problem is compounded by the fact that relatively few researchers can actually study the IMF with the same definition, making it difficult for applying the CEEMD and comparison between studies. The time complexity of CEEMD belongs to non-deterministic polynomial time and it is insufficient for real-time processing. The potential applications of CEEMD merit more studies under standardized parameter setting, including $1 / 15$ added white noise ratio, 200 ensemble number, and 0.2 as the value of stop criterion of the sifting process.

\section{Conclusion}

This paper demonstrates useful methods to improve the reliability of CEEMD of ABP signal. The characteristics of IMFs provides information about cardiac rhythm, intrinsic waveform mophology of $\mathrm{ABP}$ signal, and intrinsic influence of respiration fluctuations of $\mathrm{ABP}$ signal during different respiratory patterns. The mono-components extraction of $\mathrm{ABP}$ in adaptive time scale might help to discover the optimal frequency band of different physiological mechanisms. The CEEMD of ABP signal is able to provide important physiologic information without simultaneous ECG and respiratory monitoring. This study might serve as a guide to analyze specific IMFs components for $\mathrm{ABP}$ signal, and to evaluate the intrinsic characteristics of $\mathrm{ABP}$ signal qualitatively and quantitatively by averaged period and energy density. The proposed method helps for the researches on applications of CEEMD in pulse diagnosis and the exploration of optimal frequency band of physiological control process.

\section{Acknowledgements}

This work was fully supported by the Taiwan Ministry of Science and Technology under grant numbers MOST-103-2220-E-009-006, MOST-103-2221-E-009-139 and MOST-103- 2218-E-009-016 and in part by 'Aim for the Top University Plan' of the National Chiao Tung University and Ministry of Education, Taiwan, R.O.C.
This work was also supported in part by the UST-UCSD International Center of Excellence in Advanced Bioengineering sponsored by the Taiwan National Science Council I-RiCE Program under grant number NSC-101-2911-I-009-101.

\section{References}

1. Toorop GP, Westerhof N, Elzinga G (1987) Beat-to-beat estimation of peripheral resistance and arterial compliance during pressure transients. Am J Physiol 252: H1275-1283.

2. Rietzschel ER, Boeykens E, De Buyzere ML, Duprez DA, Clement DL (2001) A comparison between systolic and diastolic pulse contour analysis in the evaluation of arterial stiffness. Hypertension 37: E15-22.

3. Miyakawa K, Koepcen HP, Polosa C (1984) Mechanism of Blood Pressure Wave, Tokyo: Japan Scientific Societies Press, Springer-Verlag, 257-275.

4. Andriessen P, Schoffelen RL, Berendsen RC, de Beer NA, Oei SG, et al (2004) Noninvasive assessment of blood pressure variability in preterm infants. Pediatr Res 55: 220-223.

5. Jong WD (2000) Blood Pressure Variability in Neonates: With a Specia Focus on Signal Acquisition and Signal Processing, Technische Universiteit Eindhoven, 12-14.

6. Mukkamala R, Reisner AT, Hojman HM, Mark RG, Cohen RJ (2006) Continuous cardiac output monitoring by peripheral blood pressure waveform analysis. IEEE Trans Biomed Eng 53: 459-467.

7. Parati G, Saul JP, Di Rienzo M, Mancia G (1995) "Spectral Analysis of Blood Pressure and Heart Rate Variability in Evaluating Cardiovascular Regulation. A Critical Appraisal," Hypertension 25(6): 1276-1286.

8. Aboy M, McNames J, Thong T, Tsunami D, Ellenby MS, et al. (2005) An automatic beat detection algorithm for pressure signals. IEEE Trans Biomed Eng 52: 1662-1670.

9. Li BN, Dong MC, Vai MI (2010) "On an Automatic Delineator for Arterial Blood Pressure," Biomed. Signal Process Control 5(1): 76-81.

10. La Rovere MT, Pinna GD, Raczak G (2008) Baroreflex sensitivity: measurement and clinical implications. Ann Noninvasive Electrocardiol 13: 191-207.

11. Barbieri R, Triedman JK, Saul JP (2002) Heart rate control and mechanica cardiopulmonary coupling to assess central volume: a systems analysis. Am J Physiol Regul Integr Comp Physiol 283: R1210-1220.

12. Deschamps A, Kaufman I, Backman SB, Plourde G (2004) "Autonomic Nervous System Response to Epidural Analgesia in Laboring Patients by Wavelet Transform of Heart Rate and Blood Pressure Variability," Anesthesiology 101(1): 21-27

13. (1996) Task force of the European Society of Cardiology and the North American Society of pacing and Electrophysiology, "Heart Rate Variability Standards of Measurement, Physiological Interpretation, and Clinical Use," Circulation 93: 1043-1065.

14. Clayton RH, Bowman AJ, Ford GA, Murray A (1995) Measurement of baroreflex gain from heart rate and blood pressure spectra: a comparison of spectral estimation techniques. Physiol Meas 16: 131-139.

15. Omboni S, Parati G, Castiglioni P, Di Rienzo M, Imholz BP, et al. (1998) Estimation of blood pressure variability from 24-hour ambulatory finger blood pressure. Hypertension 32: 52-58.

16. Huang NE, Shen Z, Long SR, Wu MC, Shih HH, et al. (1998) "The Empirical Mode Decomposition and the Hilbert Spectrum for Nonlinear and NonStationary Time Series Analysis," Proc R Soc Lond A 454: 903-995.

17. Huang NE, Wang G, Chen XY, Qiao F, Wu Z (2010) "On Intrinsic Mode Function." Adv Adapt Data Anal 2(3): 277-293.

18. Wu MC, Huang NE (2009) "Biomedical data processing using HHT: A Review," Advance Biosignal Processing, Amine NAIT-ALI Ed., Chapter 19, Springer, 335-352.

19. Wu Z, Huang NE (2004) "A Study of the Characteristics of White Noise Using the Empirical Mode Decomposition Method." Proc R Soc Lond A 460: 15971611.

20. Flandrin P, Rilling G, Goncalves $P$ (2004) "Empirical Mode Decomposition as a Filter Bank," IEEE Signal Processing Lett 11: 112-114.

21. Agarwal V, Tsoukalas LH (2007) "Denoising Electrical Signal via Empirical Mode Decomposition," Proceeding of iREP Symposium- Bulk Power System Dynamics and Control- VII, Revitalizing Operational Reliability, Charleston, SC, 


\section{USA, Aug. 19-24}

22. Chang KM, Liu SH (2011) "Gaussian Noise Filtering from ECG by Wiener Filter and Ensemble Empirical Mode Decomposition," Journal of Signal Processing Systems 64: 249-264.

23. Wu Z, Huang NE (2009) "Ensemble Empirical Mode Decomposition: A Noise Assisted Data Analysis Method," Adv Adapt Data Anal 1: 1-42.

24. Karagiannis A, Constantinou $P$ (2011) Noise-assisted data processing with empirical mode decomposition in biomedical signals. IEEE Trans Inf Technol Biomed 15: 11-18.

25. Li H, Kwong S, Yang L, Huang D, Xiao D (2011) Hilbert-Huang transform for analysis of heart rate variability in cardiac health. IEEE/ACM Trans Comput Biol Bioinform 8: 1557-1567.

26. Zhang Q, Shi Y, Teng D, Dinh A, Ko SB, et al. (2009) "Pulse Transit Time based Blood Pressure Estimation Using Hilbert Huang Transform," Proceeding of 31st Annual International Conference of the IEEE EMBS Minneapolis, Minnesota, USA, Sep. 2-6.

27. Wu HT, Lee CH, Liu AB, Chung WS, Tang CJ, et al. (2011) "Arterial Stiffness Using Radial Arterial Waveforms Measured at the Wrist as an Indicator of Diabetic Control in the Elderly." IEEE Trans Biomed Eng 58 (2): 243-252.

28. Yeh JR, Shieh JS, Huang NE (2010) "Complementary Ensemble Empirical Mode Decomposition: a Novel Noise Enhanced Data Analysis Method." Adv Adapt Data Anal 2: 135-156.

29. Yang Z, Yang L, Qing C (2010) "An Oblique-Extrema-Based Approach for Empirical Mode Decomposition.” Digit Signal Process 20: 699-714.

30. Rilling G, Flandrin P, Gonçalvès $P$ (2003) "On Empirical Mode Decomposition and Its Algorithm," In Proceedings of the 6th IEEE/EURASIP Workshop on Nonlinear Signal and Image Processing (NSIP'03), Grado, Italy, Jun 8-11.

31. Wu Z, Schneider EK, Hu ZZ, Cao L (2001) "The Impact of Glocal Warming on ENSO Variability in Climate Records," Center of Ocean Land Atmosphere Studies, COLA Technical Report 110.

32. Lo MT, Hu K, Liu Y, Peng CK, Novak V (2008) Multimodal Pressure Flow Analysis: Application of Hilbert Huang Transform in Cerebral Blood Flow Regulation. EURASIP J Adv Signal Process 2008: 785243.

33. Balocchi R, Menicucci D, Santarcangelo E, Sebastiani L, Gemignani A et al. (2004) "Deriving the Respiratory Sinus Arrhythmia from the Heartbeat Time Series using Empirical Mode Decomposition," Chaos Solitons Fractals, 20 171-177.

34. Chen CS, Liu CC, Wu HT Liu AB (2011) "Ensemble Empirical Mode Decomposition for Atherosclerosis in High-Risk Subjects," Proceeding of the 8th International Conference on Information, Communications and Signal Processing (ICICS'11), Singapore, Dec. 13-16.

35. Li JK, Zhu Y, Geipel PS (2010) Pulse pressure, arterial compliance and wave reflection under differential vasoactive and mechanical loading. Cardiovasc Eng 10: 170-175.

36. Jing J, Hu YH, Li X, Huang Z (2007) "Feature Extraction of Pulse Signal Based on Hilbert-Huang Transform and Singular Value Decomposition," Proceeding of 1st International Conference on Bioinformatics and Biomedical Engineering (ICBBE'07), Wuhan, China, July 6-8, 1007-1010.

37. De Burgh Daly M (2011) "Interactions between Respiration and Circulation," Compr. Physiol 529-594.

38. Zh A Donina (2011) "Intersystem Relationship between Respiration and Blood Circulation," Hum Physiol 37(2): 229-239.

39. Sin PY, Galletly DC, Tzeng YC (2010) Influence of breathing frequency on the pattern of respiratory sinus arrhythmia and blood pressure: old questions revisited. Am J Physiol Heart Circ Physiol 298: H1588-1599. 